\title{
进 展
}

\section{嫦娥五号样品揭示月球20亿年前火山活动 及源区性质}

\author{
刘守偈 ${ }^{1}$, 周琴 ${ }^{2}$, 李秋立 ${ }^{3}$, 胡森 ${ }^{3}$, 杨蔚 $^{3 *}$ \\ 1. 中国地质科学院地质研究所, 北京 100037 ; \\ 2. 中国科学院国家天文台, 北京 100101; \\ 3. 中国科学院地质与地球物理研究所, 北京 100029 \\ *通讯作者, E-mail: yangw@mail.iggcas.ac.cn
}

收稿日期: 2021-11-03; 收修改稿日期: 2021-11-10; 接受日期: 2021-11-16; 网络版发表日期: 2021-11-19

\begin{abstract}
摘要 嫦娥五号任务实现使人类时隔44年再次采回新的月球样品, 也是中国首次地外天体采样. 近期, 中国地质 科学院地质研究所、中国科学院国家天文台和中国科学院地质与地球物理研究所的研究团队先后报道了嫦娥五 号样品的首批研究成果. 嫦娥五号月壤的基础物性参数均与Apollo和Luna月壤样品的变化范围一致, 但嫦娥五号 月壤的粒度更细, 分选更好, 真密度略微低于月海玄武岩。对嫦娥五号月壤中玄武岩岩屑的富U矿物定年结果显 示, 其形成于 20 亿年前, 较此前获得的最年轻月球样品(28亿年)还要年轻 8 亿年, 确证了月球火山活动至少可以持 续至20亿年前. 原位 Sr-Nd同位素分析表明, 嫦娥五号玄武岩源自亏损的月幔, 克里普组分的贡献不超过 $5 \%$, 从而 排除了源区富含放射性生热元素提供额外热源使得月幔熔融的假说. 同时, 嫦娥五号玄武岩磷灰石和熔体包裹体 的水含量和氢同位素分析表明, 其源区也不富含水, 因而不能降低熔点导致月幔熔融. 因此, 月球火山活动持续如 此之久的原因仍然不清楚, 这也为未来月球探测和研究提供了新的方向.
\end{abstract}

关键词 嫦娥五号, 月壤, 玄武岩, $\mathrm{Pb}-\mathrm{Pb}$ 定年, $\mathrm{Sr}-\mathrm{Nd}$ 同位素, 水, $\mathrm{H}$ 同位素

\section{1 引言}

1969 1976年间，美国Apollo计划和苏联Luna计划 分别完成6次载人和 3 次无人月球采样返回任务，为人 类提供了研究月球矿物组成、化学成分、形成年龄等 信息的直接样品. 这些样品的研究奠定了月球形成和 演化大框架，同时揭示地球和月球的内在成因联系， 极大地促进了行星科学的发展. 时隔44年，2020年12 月17日, 中国嫦娥五号返回器成功着陆于内蒙古四子
王旗, 带回了 $1731 \mathrm{~g}$ 月球样品, 这是中国首次完成地外 天体采样 (Yang和Lin, 2021). 嫦娥五号工程任务圆满 成功, 但是它的科学目标(Zhou等, 2021) 的实现, 还取 决于样品的科学研究.

嫦娥五号的着陆点 $\left(51.92^{\circ} \mathrm{W}, 43.06^{\circ} \mathrm{N}\right)(\mathrm{Wang}$ 等, 2021)远离美国Apollo计划和苏联Luna计划的采样区. 撞击坑统计定年结果显示嫦娥五号着陆点的熔岩流异 常年轻, 仅20 12亿年(Wu等, 2018; Jia等, 2020; Qian 等，2021)，显著年轻于Apollo和Luna计划返回的玄武 
岩(39 31亿年). 对嫦娥五号月壤样品中玄武岩岩屑的 同位素定年, 不仅可以精确厘清着陆区的地质年龄, 而 且可以标定月球撞击通量曲线，从而提高撞击坑年代 学在10 30亿年之间定年精度. 此外, 岩石地球化学研 究可以揭示月球年轻火山活动的成因和性质，从而深 入理解晚期月球内部物质组成和热演化历史.

最近, 中国地质科学院地质研究所、中国科学院 国家天文台和中国科学院地质与地球物理研究所的研 究团队先后报道了嫦娥五号月壤样品的首批研究成果 (Che等, 2021; Hu等, 2021; Li C L等, 2021; Li Q L等, 2021; Tian等, 2021). 这些成果解答了嫦娥五号月壤样 品的物理化学性质、嫦娥五号月海玄武岩的形成时代 和源区性质等问题，为未来月球探测和研究提供了新 方向(Mitchell, 2021).

\section{2 嫦娥五号月壤的基本特征}

根据采样方式的差异, 嫦娥五号月球样品分为铲 取样 (CE5C)和钻取样(CE5Z)两类. 其中, 铲取样在分 样处理之前已经被充分混合，整体为灰黑色，粒度较 细, 重量大约 $1480 \mathrm{~g}$. 中国科学院国家天文台团队针对 铲取样，开展了基础物性参数、颗粒特征、矿物种类 和化学成分等方面的分析( $\mathrm{Li} \mathrm{C} \mathrm{L}$ 等, 2021).

利用光学显微镜，对 $155 \mathrm{mg}$ 充分分散的铲取月壤 颗粒进行显微光学成像. 通过对图像中单颗粒的几何 尺寸测量和统计获得，95\%数量的月壤颗粒粒径分布

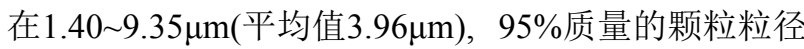
分布在 $4.84 \sim 432.27 \mu \mathrm{m}$ (平均值 $49.80 \mu \mathrm{m}$ ). 利用密度分 析仪和惰性气体吸附比表面积分析仪获得铲取月壤的
堆密度为 $1.2387 \mathrm{~g} \mathrm{~cm}^{-3}$, 真密度为 $3.1952 \mathrm{~g} \mathrm{~cm}^{-3}$, 比表面 积为 $0.56 \mathrm{~m}^{2} \mathrm{~g}^{-1}$. 这些基础物性参数均与Apollo和Luna 月壤样品的变化范围一致，但嫦娥五号月壤的粒度更 细, 分选更好, 真密度略微低于月海玄武岩(Carrier III 等, 1991; Graf, 1993).

利用X射线衍射分析仪, 对 $100 \mathrm{mg}$ 月壤的物相类型 及含量进行了分析, 识别出斜长石 $(30.1 \%)$ 、普通辉石 $(30.9 \%)$ 、易变辉石 $(11.1 \%)$ 、铁橄榄石 $(4.1 \%)$ 、镁橄 榄石 $(1.6 \%)$ 、钛铁矿 $(4.5 \%)$ 、磷灰石 $(0.7 \%)$ 、石英 $(0.4 \%)$ 和非晶质玻璃 $(16.6 \%)$. 与Apollo月海和高地样 品相比, 嫦娥五号样品中辉石含量相对较高, 斜长石 含量相对较低, 属于典型的月海玄武岩矿物组成. 利 用中子活化分析和X射线苂光光谱仪技术，对嫦娥五 号月壤进行了化学成分分析. 与Apollo和Luna样品相 比，嫦娥五号月壤的 $\mathrm{Al}_{2} \mathrm{O}_{3}(10.8 \%)$ 和 $\mathrm{CaO}(11 \%)$ 含量较 低, $\mathrm{FeO}(22.5 \%)$ 含量较高, 明显远离斜长质高地和克 里普(KREEP)端元, 靠近月海玄武岩端元(图1).

\section{3 嫦娥五号玄武岩的岩石地球化学特征}

嫦娥五号月壤样品中的较大颗粒主要是玄武岩岩 屑、凝结集块岩、角砾岩和玻璃等四种类型 $(\mathrm{Li} \mathrm{C} \mathrm{L}$ 等, 2021). 基于岩相学研究(Che等, 2021; Li C L等, 2021; Tian等, 2021), 玄武岩岩屑主要可以划分为斑状 结构、辉绿/次辉绿结构、嵌晶结构、等粒结构等 (图2).

嫦娥五号玄武岩主要矿物包括单斜辉石、斜长 石、橄榄石和钛铁矿，次要矿物包括尖晶石、钾长 石、磷灰石等，副矿物包括斜锆石、钙钛锆石、静海

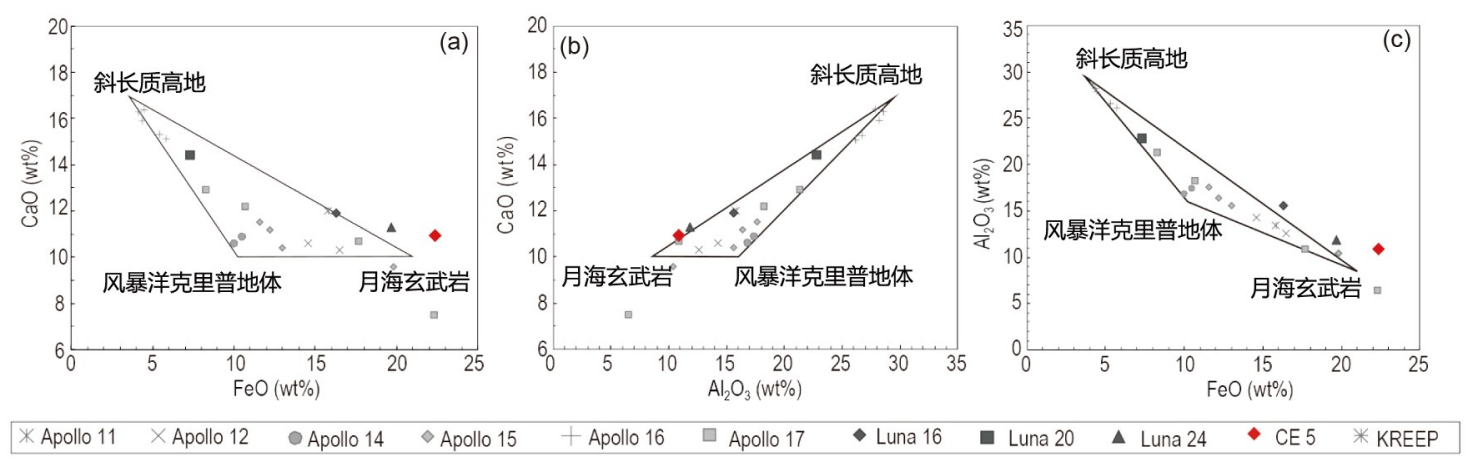

图 1 (网络版彩图)嫦娥五号月壤与阿波罗和月球号样品的化学成分对比图

(a) (c) 月壤的 $\mathrm{Al}_{2} \mathrm{O}_{3} 、 \mathrm{CaO}$ 和 $\mathrm{FeO}$ 元素相关图, 底图及数据来自Lucey等(2006). 图修改自 Li C L 等(2021) 

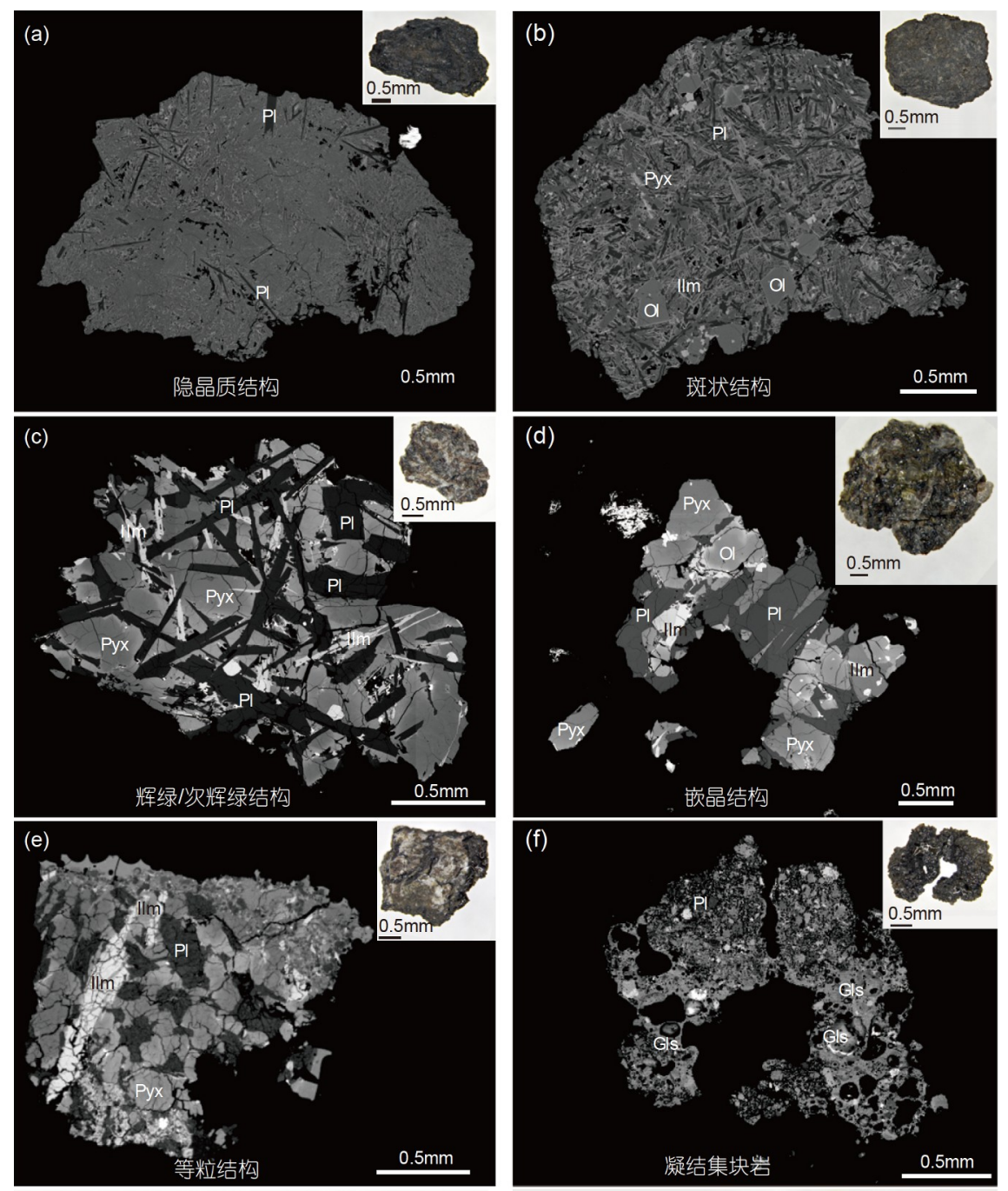

(g)

(h)
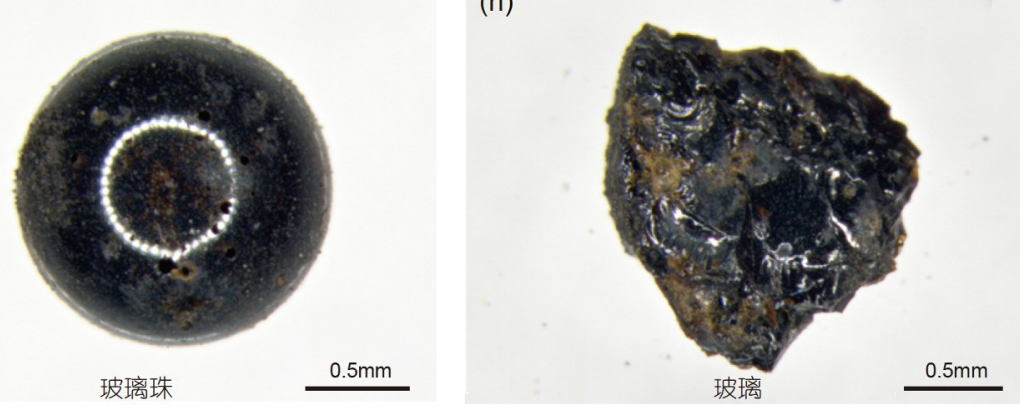

图 2 (网络版彩图)嫦娥五号月壤中典型的玄武质岩屑、凝结集块岩和玻璃的背散射电子图像和体视显微镜照片 (a) (f) 是不同结构的玄武岩和凝结集块岩的背散射照片, 背散射照片的右上角是对应颗粒的体视显微镜照片. (g) (h) 是玻璃的体视显微镜照 片. 矿物缩写: Pyx, 辉石; Pl, 斜长石; Ol, 橄榄石; Ilm, 钣铁矿; Gls, 玻璃. 图修改自Li C L等(2021)

石、白磷钙矿等(Che等, 2021; Li C L 等, 2021; Tian等, 2021). 斜长石的An值变化范围在75.0 95.5, 大部分为 倍长石(平均组成 $\mathrm{An}_{83.9} \mathrm{Ab}_{15.2} \mathrm{Or}_{0.9}$ ); 辉石主要以普通辉
石为主，成分变化较大，平均组成为 $\mathrm{Wo}_{32.9} \mathrm{En}_{28.2} \mathrm{Fs}_{38.9}$, 易变辉石较少; 橄榄石的成分变化较大(Fo值1.0 58.3, 平均值为 37.2), 其中大部分橄榄石的Fo值小于 50 . 

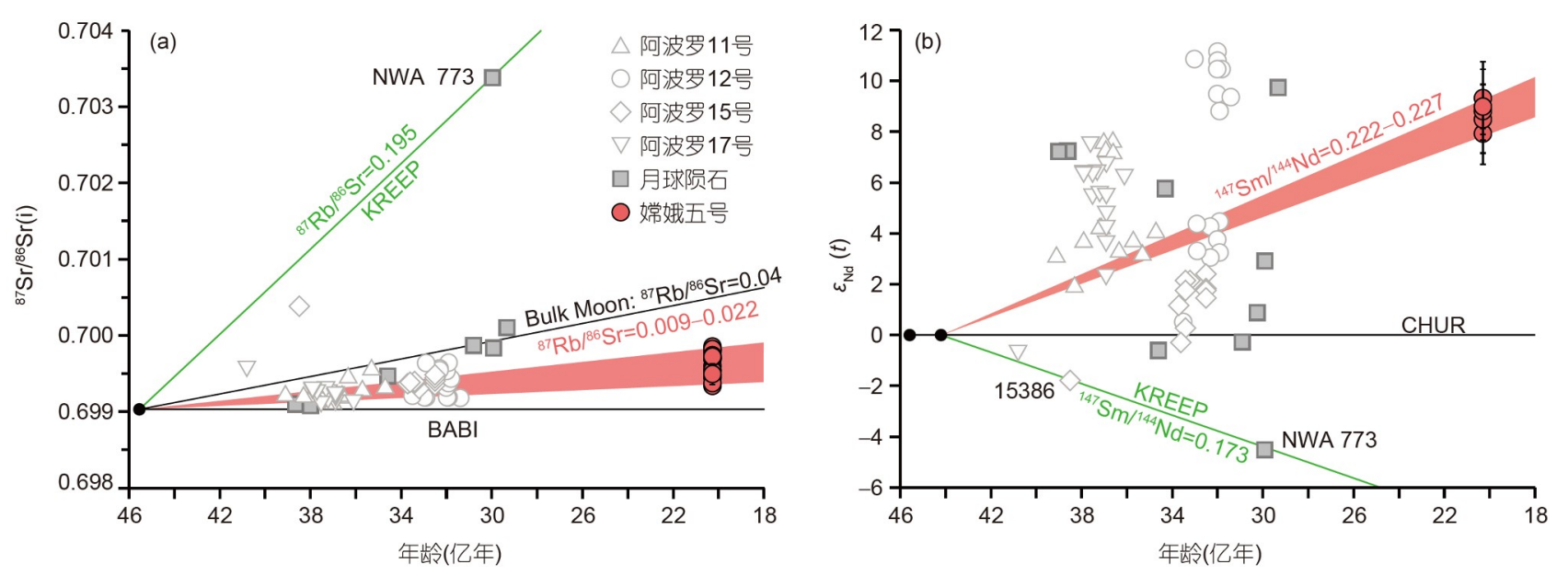

图 3 (网络版彩图)嫦娥五号玄武岩Sr-Nd同位素特征

嫦娥五号玄武岩 $\mathrm{Sr}-\mathrm{Nd}$ 同位素与克里普具有显著的差异, 计算表明, 克里普组分的贡献不足 $0.5 \%$. 修改自 Tian等 $(2021)$

所有玄武岩岩屑的辉石中 $\mathrm{Ti}$ 和 $\mathrm{Al}$ 的摩尔含量符合 $1: 2$ 的相关性, 说明这些玄武岩可能源自同一次火山熔 岩流，其矿物粒径和结构差异反映的是熔岩流不同位 置冷却速率的差异. 由此, Tian等(2021)估算出嫦娥五 号玄武岩的平均成分，其 $\mathrm{TiO}_{2}$ 含量 $(5.7 \pm 1.9) \mathrm{wt} . \%$, $\mathrm{Al}_{2} \mathrm{O}_{3}$ 含量 $(11.6 \pm 1.9) w t . \%, \mathrm{~K}_{2} \mathrm{O}$ 含量 $(0.1 \pm 0.1) \mathrm{wt} . \%$, 属于低 $\mathrm{Ti} /$ 高 $\mathrm{Al} /$ 低K类型(Neal和Taylor， 1992). Che 等(2021)估算出类似的平均成分, 但 $\mathrm{TiO}_{2}$ 的含量稍高 (6 8wt.\%). 相比于Apollo和Luna低钛玄武岩，嫦娥 五号玄武岩具有较高的 $\mathrm{FeO} 、 \mathrm{TiO}_{2} 、 \mathrm{Al}_{2} \mathrm{O}_{3}$ 含量和较 低的 $\mathrm{Mg}^{\#}(32.1)$. 此外, 嫦娥五号玄武岩具有类似于克 里普岩(KREEP)的轻稀土富集(球粒陨石的150倍)和 高Th含量 $\left(4.5 \mathrm{ppm}, 1 \mathrm{ppm}=1 \mu \mathrm{g} \mathrm{\textrm {g } ^ { - 1 }}\right.$ ) 的特征(Tian等, 2021).

斜长石的 $\mathrm{Sr}$ 和白磷钙矿的 $\mathrm{Nd}$ 同位素分析结果显 示, 嫦娥五号玄武岩具有非常低的初始 ${ }^{87} \mathrm{Sr} /{ }^{86} \mathrm{Sr}$ 比值 (0.69934 0.69986)和亏损的 $\varepsilon_{\mathrm{Nd}}(t)$ 值(7.9 9.3), 与克里 普岩存在显著的差异(图3). 计算表明，嫦娥五号玄武 岩的源区中, 克里普组分的贡献不足 $0.5 \%$ (Tian等, 2021). Che等(2021)和Li Q L等(2021)也根据初始Pb组 成计算出玄武岩源区的 $\mu$ 值，结果分别为 $677 \pm 3$ 和 684 \pm 40 . 这一源区 $\mu$ 值显著不同于克里普组分特征，进一 步支持嫦娥五号玄武岩源区没有克里普组分的贡献. 嫦娥五号玄武岩类似于克里普岩的稀土元素特征来自 于其经历的小比例 $(2 \sim 3 \%)$ 部分熔融和强烈的 (43 88\%)分离结晶作用(Tian等, 2021).

\section{4 嫦娥五号玄武岩的形成时代}

中国地质科学院地质研究所和中国科学院地质与 地球物理研究所的团队分别对嫦娥五号月壤中的玄武 岩岩屑开展了离子探针原位定年(Che等, 2021; Li Q L 等, 2021). Che等(2021)对两个岩屑共计50个不同区域 进行了 $\mathrm{Pb}-\mathrm{Pb}$ 定年分析, 分析束斑直径约为 $7 \mu \mathrm{m}$, 主要 包括磷酸盐颗粒、含 $\mathrm{Ba}$ 钾长石颗粒、富 $\mathrm{K}$ 玻璃以及富 $\mathrm{Zr}$ 矿物区域. 数据处理则采用 $\mathrm{Pb}-\mathrm{Pb}$ 等时线法 (Snape

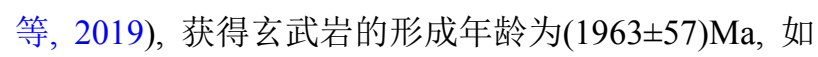
仅考虑含锆矿物, 给出的年龄为 $(2011 \pm 50) \mathrm{Ma}$ (图4). Li Q L 等(2021)对全部四种类型的玄武岩岩屑进行了 Pb$\mathrm{Pb}$ 定年分析. 对每一种结构玄武岩进行含锆矿物的定 位和分析, 结合主要造岩矿物, 分别构建 $\mathrm{Pb}-\mathrm{Pb}$ 等时线, 四条等时线斜率一致, 获得四个在误差范围内一致的 $\mathrm{Pb}-\mathrm{Pb}$ 年龄, 进一步支持了嫦娥五号月壤中的玄武岩岩 屑都来自同一次熔岩流. 统计分析的 47 个岩屑的结果, 最重要的是其中的 51 颗含锆矿物, 即斜锆石、钙钛锆 石、静海石精确限定了玄武岩喷发时代为 $(2030 \pm 4)$ $\mathrm{Ma}$ (图4). 这类矿物一则具有高U含量和非常低的初始 $\mathrm{Pb}$ 含量, 二则是玄武岩的结晶矿物, 因此被公认为玄武 岩定年最具代表性的矿物.

嫦娥五号玄武岩的精确年龄为撞击坑定年曲线提 供了关键的针点. 此前不同研究者通过撞击坑统计定 年法得到的嫦娥五号着陆区岩石年龄相差很大 $(\mathrm{Wu}$ 等, 2018; Jia等，2020; Qian等，2021), 最主要的原因是 

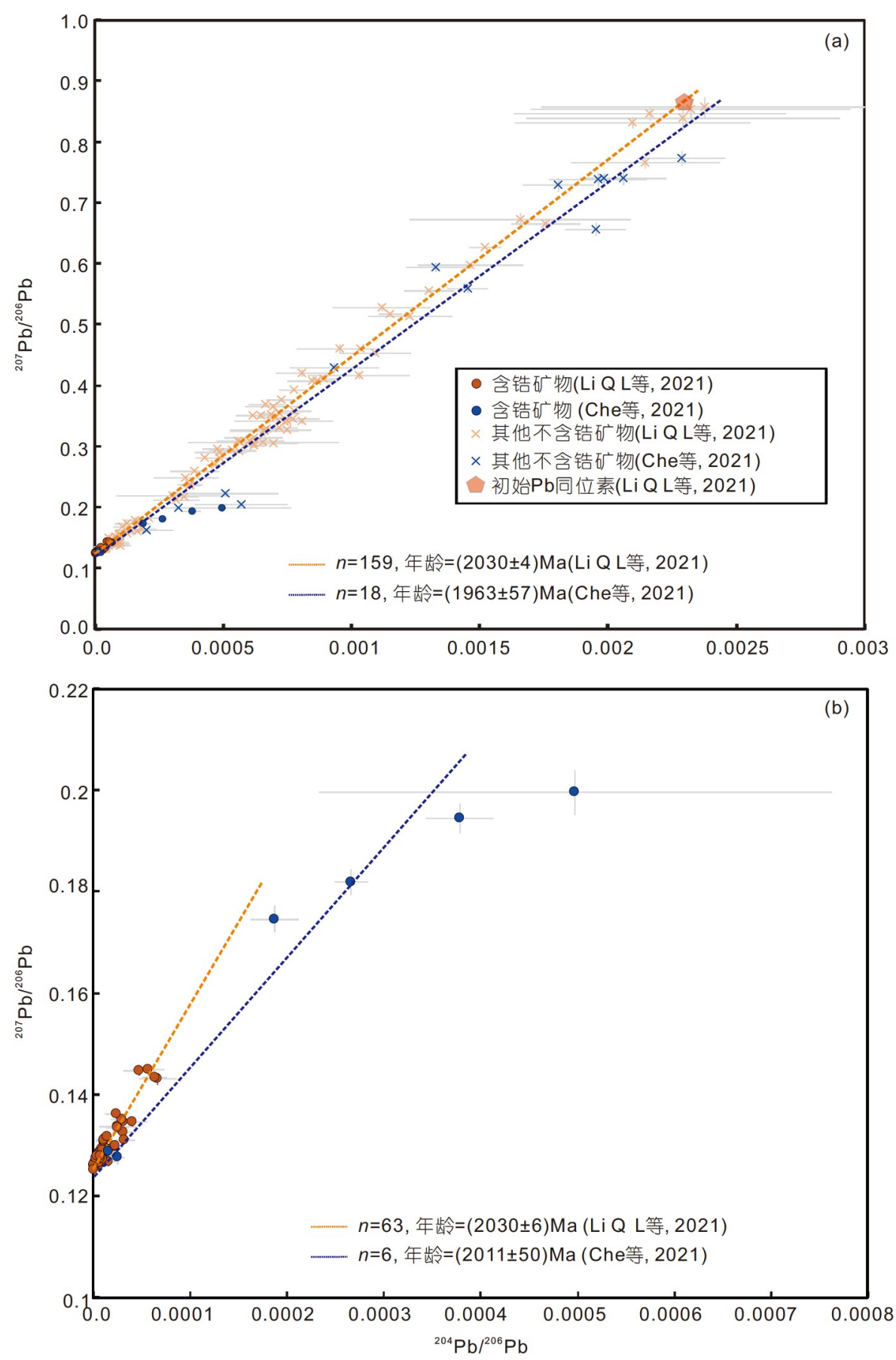

图 4 (网络版彩图)嫦娥五号玄武岩岩屑Pb-Pb等时线

(a) 多矿物(包括含铅矿物和主要造岩矿物) $\mathrm{Pb}-\mathrm{Pb}$ 等时线; (b) 含锆矿物(包括斜锆石、钻铁锆石、静海石) $\mathrm{Pb}-\mathrm{Pb}$ 等时线. 数据源自 Che等(2021)和 Li Q L等(2021)

10 30亿年之间没有定标点(图5). 嫦娥五号玄武岩年 龄将极大提高“撞击坑定年法”的精度，为未来研究内 太阳系行星表面年龄打下扎实基础.

\section{5 嫦娥五号玄武岩的源区水含量}

为揭示嫦娥五号玄武岩源区的水含量，中国科学 院地质与地球物理研究所团队借助纳米离子探针对 


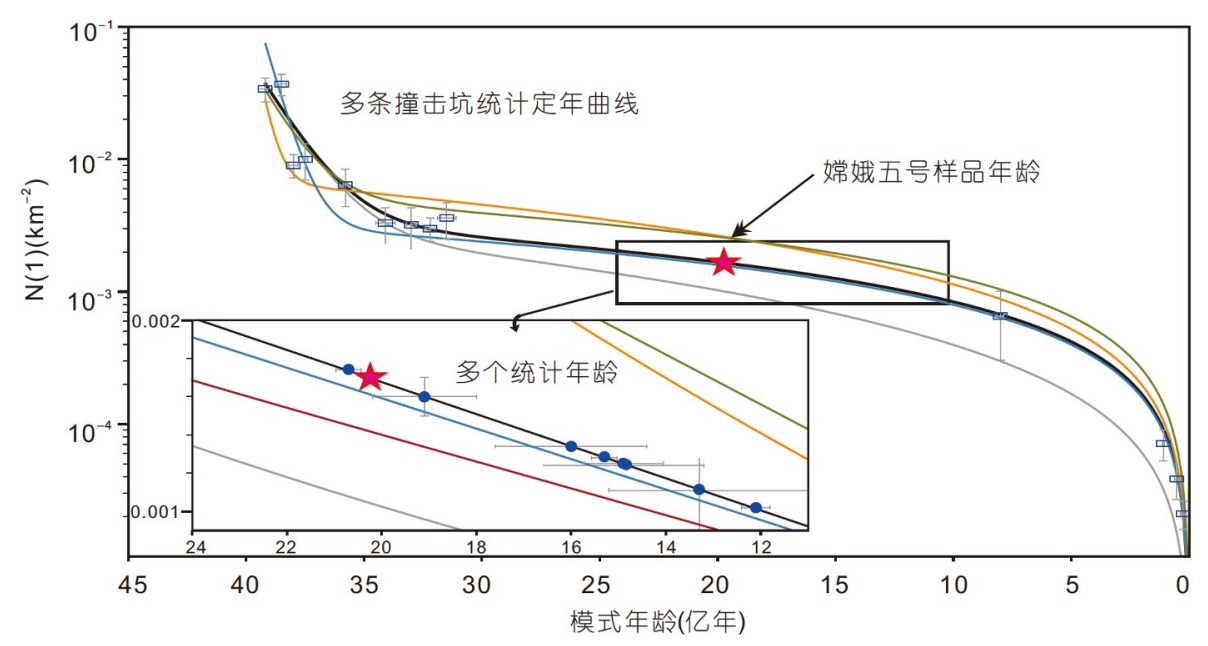

图 5 (网络版彩图)嫦娥五号玄武岩年龄在撞击坑统计定年曲线上的针点作用(修改自 Li Q L 等, 2021)
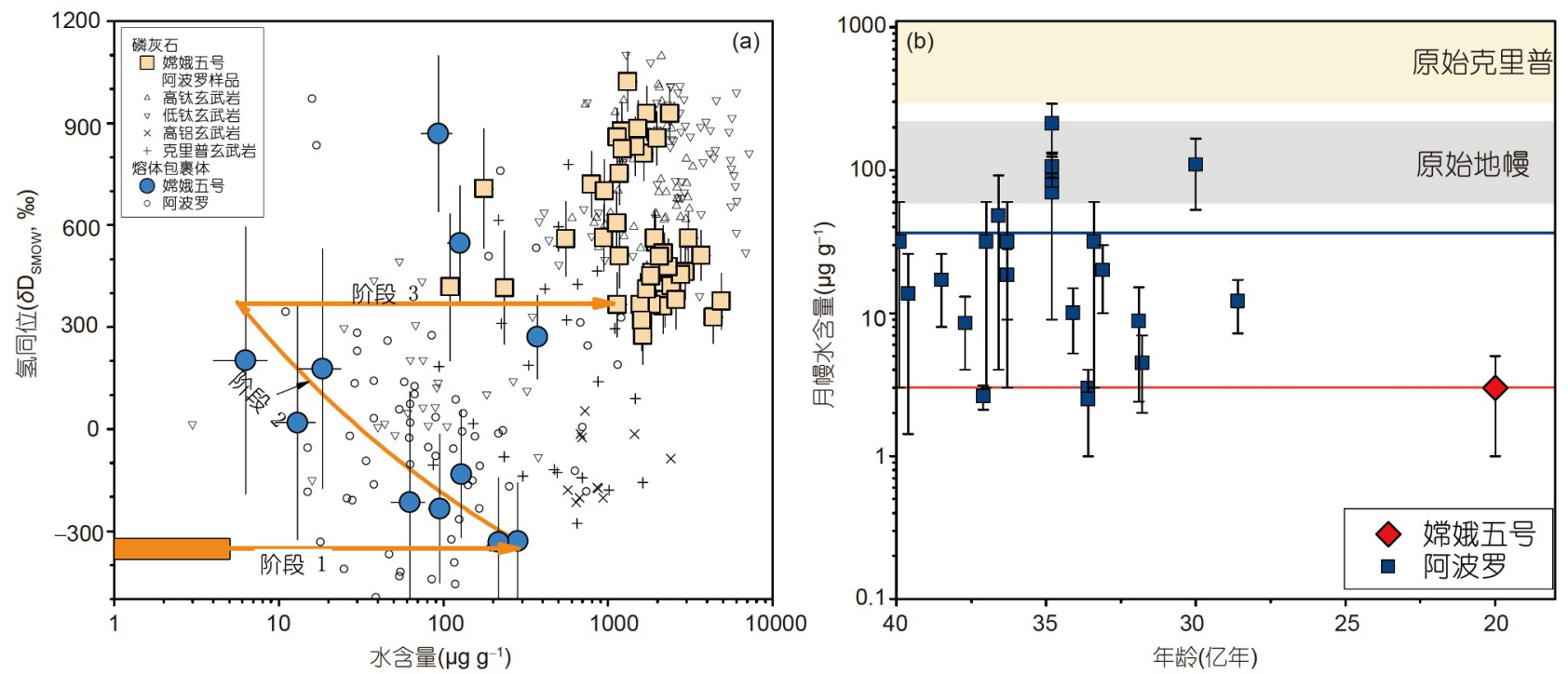

图 6 (网络版彩图)嫦娥五号玄武岩样品中磷灰石和熔体包裹体的水含量和氢同位素组成(a)以及月幔水含量随时间演化(b) 大部分熔体包裹体的水含量和氢同位素组成具有明显的负相关, 指示岩浆去气过程. 磷灰石明显富集重的氢同位素, 经历过强烈的去气, 与其 晚期结晶的岩相特征相符. 嫦娥五号玄武岩的熔体包裹体和磷灰石水含量和氢同位素的相关性, 记录了岩浆不同阶段的演化过程. 该过程可 以划分为三个阶段, 阶段 1 : 月幔部分熔融形成嫦娥五号的岩浆; 阶段 2 : 岩浆侵入或溢流, 被铁铁矿捕获; 阶段 3 : 岩浆溢流到月表后发生强烈的 去气丢失导致氢同位素分馏, 最后形成磷灰石, 岩浆完全固化成岩. 修改自Hu等(2021)

嫦娥五号玄武岩中的熔体包裹体和磷灰石开展了水 含量和氢同位素分析(Hu等，2021)。熔体包裹体是矿 物在岩浆结晶过程中捕获的岩浆样品. 分析结果表 明, 包裹在钛铁矿中的熔体包裹体, $\mathrm{D} / \mathrm{H}$ 比很低, 并与 水含量呈负相关，说明不同包裹体实际上代表了岩浆 在整个喷发和结晶过程不同阶段采集的样本(图6a). 最低 $\mathrm{D} / \mathrm{H}$ 比值的包裹体，可能没有经历去气作用或者
去气作用影响非常小，因而含有最原始岩浆的 $\mathrm{D} / \mathrm{H}$ 比 值. 磷灰石是月球样品中唯一的含水矿物. 嫦娥五号 样品中的磷灰石都分布在辉石、长石、钛铁矿、橄 榄石等主要组成矿物粒间, 说明它们是岩浆结晶晚期 形成的，从而记录了岩浆结晶晚期阶段的水及其同位 素组成. 嫦娥五号磷灰石中的水具有高的 $\mathrm{D} / \mathrm{H}$ 比值, 指 示了岩浆在上升喷出过程中, 水以氢气的形式发生过 
丢失，造成残余岩浆中的水具有重的氢同位素组成, 与岩相特征相符.

最早被捕获熔体包裹体 $(\mathrm{D} / \mathrm{H}$ 比最低), 含有最原始 岩浆的 $\mathrm{D} / \mathrm{H}$ 比值, 其水含量约为 $280 \mu \mathrm{g} \mathrm{g}^{-1}$. 另外, 根据 磷灰石的水含量和氢同位素组成，假定嫦娥五号玄武 岩的水都赋存在磷灰石中，经过校正去气丢失效应后， 估算原始岩浆的水含量与岩浆包裹体的测定值在误差 范围内一致. 月海玄武岩是月幔部分熔融结晶形成的 岩石. 岩石成因研究表明嫦娥五号玄武岩是从月幔部 分熔融(2 3\%)，再经过结晶分异(43 88\%)形成(Tian 等，2021). 这个过程使岩浆中的水含量提高了约 100 250倍，因此，估算出的月幔源区的水含量仅为 $1 \sim 5 \mu \mathrm{g} \mathrm{g}^{-1}$, 表明嫦娥五号玄武岩的月幔源区非常“干”. 这么低的水含量不太可能导致月幔源区发生部分熔融 形成嫦娥五号玄武岩.

嫦娥五号玄武岩月幔源区的水含量与阿波罗月岩 和月球陨石估算的月幔水含量相比偏低，落在月幔水 含量估值的最低端(图6b). 导致如此低水含量的一种 可能性是嫦娥五号着陆区的月幔经历了更长时间的岩 浆活动，导致其源区的水在部分熔融过程中被多次抽 取，使得源区水含量变低. 这个过程和嫦娥五号着陆 区长时间的火山活动一致. 当然，另一种可能性是月 幔的水含量本身非常不均一，而嫦娥五号玄武岩月幔 源区一直以来就很低.

\section{6 展望}

嫦娥五号玄武岩 20 亿年的形成年龄，较此前获得 的最年轻月球样品(28亿年)还要年轻 8 亿年, 确证了月 球火山活动至少可以持续至 20 亿年前. 这个结果也对 月球热演化历史提出了挑战, 由于月球质量较小，理 论上它的火山活动在 25 亿年前就应当结束(Spohn等, 2001). 嫦娥五号的岩石地球化学研究表明，其源区并 非富含放射性生热元素，无法提供额外的热源产生月 幔熔融. 同时, 其水含量和氢同位素分析表明, 其源区 也不富含水，因而不能降低熔点导致月幔熔融. 因此, 月球火山活动能持续如此之久的原因仍然不清楚，这 也为未来嫦娥五号月球样品的更深入的研究提供了新 的方向(Mitchell, 2021).

致谢 感谢惠鹤九教授和两位审稿人对本文初稿提出的 宝贵意见。

\section{参考文献}

Carrier III W D, Olhoeft G R, Mendell W. 1991. Physical properties of the lunar surface. In: Heiken G H, Vaniman D T, French B M, eds. Lunar Sourcebook, A User's Guide to the Moon. Cambridge: Cambridge University Press. 475-594

Che X, Nemchin A, Liu D, Long T, Wang C, Norman M D, Joy K H, Tartese R, Head J, Jolliff B, Snape J F, Neal C R, Whitehouse M J, Crow C, Benedix G, Jourdan F, Yang Z, Yang C, Liu J, Xie S, Bao Z, Fan R, Li D, Li Z, Webb S G. 2021. Age and composition of young basalts on the Moon, measured from samples returned by Chang'e-5. Science, 374: 887-890

Graf J C. 1993. Lunar soils grain size catalog. National Aeronautics and Space Administration

Hu S, He H, Ji J, Lin Y, Hui H, Anand M, Tartèse R, Yan Y, Hao J, Li R, Gu L, Guo Q, He H, Ouyang Z. 2021. A dry lunar mantle reservoir for young mare basalts of Chang'e-5. Nature, doi: 10.1038/s41586-021-04107-9

Jia M, Yue Z, Di K, Liu B, Liu J, Michael G. 2020. A catalogue of impact craters larger than $200 \mathrm{~m}$ and surface age analysis in the Chang'e-5 landing area. Earth Planet Sci Lett, 541: 116272

Li C L, Hu H, Yang M F, Pei Z Y, Zhou Q, Ren X, Liu B, Liu D, Zeng X, Zhang G, Zhang H, Liu J, Wang Q, Deng X, Xiao C, Yao Y, Xue D, Zuo W, Su Y, Wen W, Ouyang Z. 2021. Characteristics of the lunar samples returned by Chang'e-5 mission. Natl Sci Rev, doi: 10.1093/nsr/nwab188

Li Q L, Zhou Q, Liu Y, Xiao Z, Lin Y, Li J H, Ma H X, Tang G Q, Guo S, Tang X, Yuan J Y, Li J, Wu F Y, Ouyang Z, Li C, Li X H. 2021. Two billion-year-old volcanism on the Moon from Chang'e-5 basalts. Nature, doi: 10.1038/s41586-021-04100-2

Lucey P, Korotev R L, Gillis J J, Taylor L A, Lawrence D, Campbell B A, Elphic R, Feldman B, Hood L L, Hunten D, Mendillo M, Noble S, Papike J J, Reedy R C, Lawson S, Prettyman T, Gasnault O, Maurice S. 2006. Understanding the lunar surface and space-moon interactions. Rev Mineral Geochem, 60: 83-219

Mitchell R N. 2021. Chang'e-5 reveals the Moon's secrets to a longer life. Innovation, 2: 100177

Neal C R, Taylor L A. 1992. Petrogenesis of mare basalts: A record of lunar volcanism. Geochim Cosmochim Acta, 56: 2177-2211

Qian Y, Xiao L, Wang Q, Head J W, Yang R, Kang Y, van der Bogert C H, Hiesinger H, Lai X, Wang G, Pang Y, Zhang N, Yuan Y, He Q, Huang J, Zhao J, Wang J, Zhao S. 2021. China's Chang'e-5 landing site: Geology, stratigraphy, and provenance of materials. Earth Planet Sci Lett, 561: 116855

Snape J F, Nemchin A A, Whitehouse M J, Merle R E, Hopkinson T, Anand M. 2019. The timing of basaltic volcanism at the Apollo 
landing sites. Geochim Cosmochim Acta, 266: 29-53

Spohn T, Konrad W, Breuer D, Ziethe R. 2001. The longevity of Lunar volcanism: Implications of thermal evolution calculations with 2D and 3D mantle convection models. Icarus, 149: 54-65

Tian H C, Wang H, Chen Y, Yang W, Zhou Q, Zhang C, Lin H L, Huang C, Wu S T, Jia L H, Xu L, Zhang D, Li X G, Chang R, Yang Y H, Xie L W, Zhang D P, Zhang G L, Yang S H, Wu F Y. 2021. Non-KREEP origin for Chang'e-5 basalts in the Procellarum KREEP Terrane. Nature, doi: 10.1038/s41586-021-04119-5

Wang J, Zhang Y, Di K, Chen M, Duan J, Kong J, Xie J, Liu Z, Wan W, Rong Z, Liu B, Peng M, Wang Y. 2021. Localization of the Chang'e-5 lander using radio-tracking and image-based methods.
Remote Sens, 13: 590

Wu B, Huang J, Li Y, Wang Y, Peng J. 2018. Rock abundance and crater density in the candidate Chang'e-5 landing region on the Moon. J Geophys Res-Planets, 123: 3256-3272

Yang W, Lin Y. 2021. New lunar samples returned by Chang'e-5: Opportunities for new discoveries and international collaboration. Innovation, 2: 100070

Zhou C, Jia Y, Liu J, Li H, Fan Y, Zhang Z, Liu Y, Jiang Y, Zhou B, He Z, Yang J, Hu Y, Liu Z, Qin L, Lv B, Fu Z, Yan J, Wang C, Zou Y. 2021. Scientific objectives and payloads of the lunar sample return mission-Chang'e-5. Adv Space Res, doi: 10.1016/j.asr.2021. 09.001

(责任编委: 惠鹤九) 